INSTITUT NATIONAL DE RECHERCHE EN INFORMATIQUE ET EN AUTOMATIQUE

\title{
A Linear Bound on the Complexity of the Delaunay triangulation of points on polyhedral surfaces
}

\author{
Dominique Attali — Jean-Daniel Boissonnat
}

$\mathbf{N}^{\circ} 4453$

Avril 2002

THÈME 2 



\title{
A Linear Bound on the Complexity of the Delaunay triangulation of points on polyhedral surfaces
}

\author{
${\text { Dominique Attali * }{ }^{*} \text { Jean-Daniel Boissonnat }}^{\dagger}$ \\ Thème 2 - Génie logiciel \\ et calcul symbolique \\ Projet Prisme
}

Rapport de recherche $n^{\circ} 4453$ - Avril 2002 - 20 pages

\begin{abstract}
Delaunay triangulations and Voronoi diagrams have found numerous applications in surface modeling, surface mesh generation, deformable surface modeling and surface reconstruction. Many algorithms in these applications begin by constructing the three-dimensional Delaunay triangulation of a finite set of points scattered over a surface. Their running-time therefore depends on the complexity of the Delaunay triangulation of such point sets.

Although the Delaunay triangulation of points in $\mathbb{R}^{3}$ can be quadratic in the worst-case, we show that, under some mild sampling condition, the complexity of the 3D Delaunay triangulation of points distributed on a fixed number of facets of $\mathbb{R}^{3}$ (e.g. the facets of a polyhedron) is linear. Our bound is deterministic and the constants are explicitly given.
\end{abstract}

Key-words: Computational geometry, Delaunay triangulation, Voronoi diagram, polyhedral surfaces, complexity, surface sampling, surface modeling, surface reconstruction

This work has been partially supported by the ARC Costic of INRIA and by the ECG Project (Effective Computational Geometry for Curves and Surfaces) of the IST Programme of the EU (contract No IST-2000-26473).

* LIS, ENSIEG, Domaine Universitaire, BP 46, 38402 Saint Martin d'Hères Cedex. e-mail: Dominique.Attali@inpg.fr

$\dagger$ INRIA, 2004 Route des Lucioles, BP 93, 06904 Sophia-Antipolis, France. e-mail : JeanDaniel.Boissonnat@ sophia.inria.fr 


\section{Une borne linéaire sur la complexité de la triangulation de Delaunay de points distribués sur des surfaces polyédriques}

Résumé : Les triangulations de Delaunay et les diagrammes de Voronoi trouvent des applications nombreuses en modélisation de surfaces : génération de maillages, déformation, reconstruction de surfaces. Beaucoup d'algorithmes dans ce contexte commencent par construire la triangulation de Delaunay d'un ensemble fini de points pris sur la surface. Leur complexité dépend donc de la complexité de la triangulation de Delaunay des points.

La complexité de la triangulation de Delaunay de $n$ points de $R^{d}$, c'est-à-dire le nombre de ses faces, peut être $\Omega\left(n^{\left\lceil\frac{d}{2}\right\rceil}\right)$. En particulier, dans $R^{3}$, le nombre de tétraèdres peut être quadratique. Dans cet article, nous donnons une borne linéaire sur la complexité de la triangulation de Delaunay de points distribués sur un nombre fixé de facettes de $\mathbb{R}^{3}$, par exemple les faces d'un polyèdre, et vérifiant une hypothèse d'échantillonnage uniforme assez faible. Notre borne est déterministe et les constantes sont données explicitement.

Mots-clés : Géométrie algorithmique, triangulation de Delaunay, diagramme de Voronoi, surfaces polyédriques, complexité, échantillonnage de surface, modélisation de surfaces, reconstruction de surfaces 
Delaunay triangulations and Voronoi diagrams have found numerous applications in surface modeling, surface mesh generation, deformable surface modeling and surface reconstruction. Many algorithms in these applications begin by constructing the three-dimensional Delaunay triangulation of a finite set of points scattered over a surface. Their running-time therefore depends on the complexity of the Delaunay triangulation of such point sets.

Although the complexity of the Delaunay triangulation of points in $\mathbb{R}^{3}$ may be quadratic in the worst-case, we show in this paper that it is only linear when the points are distributed on a fixed number of well-sampled facets of $\mathbb{R}^{3}$ (e.g. the facets of a polyhedron). Our bound is deterministic and the constants are explicitly given.

\section{Introduction}

Delaunay triangulations and Voronoi diagrams are among the most thoroughly studied geometric data structures in computational geometry. Recently, they have found many applications in surface modeling, surface mesh generation [13], deformable surface modeling [23, 17], medial axis approximation $[4,10,22]$, and surface reconstruction $[3,1,9,2,7,6]$. Many algorithms in these applications begin by constructing the three-dimensional Delaunay triangulation of a finite set of points scattered over a surface. Their running-time therefore depends on the complexity of the Delaunay triangulation of such point sets.

It is well known that the complexity of the Delaunay triangulation of $n$ points in $\mathbb{R}^{d}$, i.e. the number of its simplices, can be $\Omega\left(n^{\left\lceil\frac{d}{2}\right\rceil}\right)$ [11]. In particular, in $\mathbb{R}^{3}$, the number of tetrahedra can be quadratic. This is prohibitive for applications where the number of points is in the millions, which is routine nowadays. Although it has been observed experimentally that the complexity of the Delaunay triangulation of well-sampled surfaces is linear (see e.g. $[9,14]$ ), no result close to this bound has been obtained yet. Our goal is to exhibit practical geometric constraints that imply subquadratic and ultimately linear Delaunay triangulations. Since output-sensitive algorithms are known for computing Delaunay triangulations [12], better bounds on the complexity of the Delaunay triangulation would immediately imply improved bounds on the time complexity of computing the Delaunay triangulation.

First results on Delaunay triangulations with low complexity have been obtained by Dwyer [15, 16] who proved that, if the points are uniformly distributed in a ball, the expected complexity of the Delaunay triangulation is only linear. Recently, Erickson $[18,19]$ investigated the complexity of three-dimensional Delaunay triangulations in terms of a geometric parameter called the spread, which is the ratio between the largest and the smallest interpoint distances. He proved that the complexity of the Delaunay triangulation of any set of $n$ points in $\mathbb{R}^{3}$ with spread $\Delta$ is $O\left(\Delta^{3}\right)$.

Despite its practical importance, the case of points distributed on a surface has not received much attention. A first result has been obtained by Golin and $\mathrm{Na}$ [20]. They proved that the expected complexity of 3D Delaunay triangulations of random points on any fixed convex polytope is $\Theta(n)$. Very recently, they extended their proof to the case of general polyhedral surfaces of $\mathbb{R}^{3}$ and obtained a $O\left(n \log ^{4} n\right)$ bound on the expected complexity of the Delaunay triangulation [21]. Deterministic bounds have also been obtained. Attali and Boissonnat [5] proved that, for any fixed polyhedral surface $S$, any so-called "light-uniform $\varepsilon$-sample" of $S$ of size $n$ has only $O\left(n^{7 / 4}\right)$ Delaunay tetrahedra. 
If the surface is convex, the bound reduces to $O\left(n^{3 / 2}\right)$. Applied to a fixed $C^{2}$ uniformly-sampled surface, the result of Erickson mentioned above shows that the Delaunay triangulation has complexity $O\left(n^{3 / 2}\right)$. This bound is tight in the worst-case. It should be noticed however that Erickson's definition of a uniform sample is rather restrictive and does not allow two points to be arbitrarily close (in which case, the spread would become infinite).

In this paper, we consider the case of points distributed on a fixed number of planar facets in $\mathbb{R}^{3}$, e.g. the facets of a given polyhedron. Under a mild uniform sampling condition, we show that the complexity of the Delaunay triangulation of the points is linear. Our bound is deterministic and the constants are explicitly given.

\section{Definitions and notations}

\subsection{Voronoi diagrams and Delaunay triangulations}

Let $E=\left\{p_{1}, \ldots, p_{n}\right\}$ be a set of points of $\mathbb{R}^{d}$. The Voronoi cell of $p_{i}$ is

$$
V\left(p_{i}\right)=\left\{x \in \mathbb{R}^{d}:\left\|x-p_{i}\right\| \leq\left\|x-p_{j}\right\| \forall j=1, \ldots, n\right\}
$$

where $\|x-y\|$ denotes the Euclidean distance between the two points $x, y$ of $\mathbb{R}^{d}$. The collection of Voronoi cells is called the Voronoi diagram of $E$, denoted $\operatorname{Vor}(E)$. The Delaunay triangulation of $E$, denoted $\operatorname{Del}(E)$ is the dual complex of $\operatorname{Vor}(E)$ (see Figure 1). If there is no sphere passing through $d+2$ points of $E, \operatorname{Del}(E)$ is a simplicial complex that can be obtained from $\operatorname{Vor}(E)$ as follows. If $E^{\prime}$ is a subset of points of $E$ whose Voronoi cells have a non empty intersection, the convex hull $\operatorname{conv}\left(E^{\prime}\right)$ is a Delaunay face and all Delaunay faces are obtained this way. It is well known that the balls circumscribing the $d$-simplices in $\operatorname{Del}(E)$ cannot contain a point of $E$ in their interior. The complexity of $\operatorname{Del}(E)$ is the number of its faces, which is also the number of faces of the dual Voronoi diagram.

A ball or a disk is said to be empty iff its interior contains no point of $E$. We also say that a sphere is empty if the associated ball is empty.

\subsection{Notations}

For a curve $\Gamma$, we denote by $l(\Gamma)$ its length. For a portion of a surface $R$, we denote by $a(R)$ its area, and by $\partial R$ its boundary. We further denote by $B(x, r)(\Sigma(x, r))$ the ball (sphere) of radius $r$ centered at $x$, and by $D_{p}(x, r)$ the disk lying in plane $P$ centered at $x \in P$ and of radius $r$.

Let $R \subset P$ be a region of $P$. The plane $P$ containing $R$ is called a supporting plane of $R$. We define:

$$
\begin{aligned}
& R \oplus_{p} \varepsilon=\left\{x \in P: D_{p}(x, \varepsilon) \cap R \neq \emptyset\right\} \\
& R \ominus_{p} \varepsilon=\left\{x \in P: D_{p}(x, \varepsilon) \subset R\right\}
\end{aligned}
$$

$R \oplus_{p} \varepsilon$ is obtained by growing $R$ by $\varepsilon$ within its supporting plane $P$ and $R \ominus_{p} \varepsilon$ is obtained by shrinking $R$ by $\varepsilon$ within its supporting plane $P$. When the supporting plane is unique or when it is clear from the context, we will simply note $R \oplus \varepsilon$ and $R \ominus \varepsilon$. 

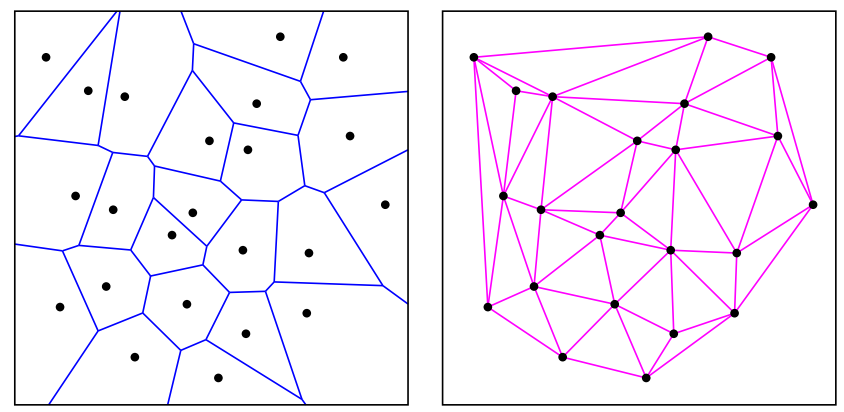

Figure 1: Voronoi diagram of a set of points on the left and its dual Delaunay triangulation on the right.

\subsection{Polyhedral surfaces}

We call polyhedral surface a finite collection of bounded polygons, any two of which are either disjoint or meet in a common edge or vertex. The polygons are called facets. Notice that we allow an arbitrary number of polygons to be glued along a common edge. In the mathematical literature, such an object is called a pure two-dimensional piece-wise linear complex. We prefer to use in this paper the term surface since surfaces are our primary concern.

In the rest of the paper, $S$ denotes an arbitrary but fixed polyhedral surface. Three quantities $C, A$ and $L$ will express the complexity of the surface $S: C$ denotes the number of facets of $S$, $A=a(S)$ its area, and $L$ the sum of the lengths of the boundaries of the facets of $S$ :

$$
L=\sum_{F \subset S} l(\partial F) .
$$

Observe that, if an edge is incident to $k$ facets, its length will be counted $k$ times.

We consider two zones on the surface, the $\varepsilon$-singular zone that surrounds the edges of $S$ and the $\varepsilon$-regular zone obtained by shrinking the facets.

Definition 1 Let $\varepsilon \geq 0$. The $\varepsilon$-regular zone of a facet $F \subset S$ consists of the points of $F$ at distance greater than $\varepsilon$ from the boundary of $F$. The $\varepsilon$-regular zone of $S$ is the union of the $\varepsilon$-regular zones of its facets. We call $\varepsilon$-singular zone of $F$ (resp. S) the set of points that do not belong to the $\varepsilon$-regular zone of $F$ (resp. $S$ ).

Observe that the $\varepsilon$-regular zone of the facet $F$ is $F \ominus \varepsilon$. The 0 -singular zone of $S$ consists exactly of the edges of $S$.

\subsection{Sample}

Any finite subset of points $E \subset S$ is called a sample of $S$. The points of $E$ are called sample points. We impose two conditions on samples. First, the facets of the surface must be uniformly sampled. Second, the sample cannot be arbitrarily dense locally. 
Definition 2 Let $S$ be a polyhedral surface. $E \subset S$ is said to be a $(\varepsilon, \kappa)$-sample of $S$ iff for every facet $F$ of $S$ and every point $x \in F$ :

- the ball $B(x, \varepsilon)$ encloses at least one point of $E \cap F$,

- the ball $B(x, 2 \varepsilon)$ encloses at most $\kappa$ points of $E \cap F$.

The 2 factor in the second condition of the definition is not important and is just to make the constant in our bound simpler. Any other constant and, in particular 1, will lead to a linear bound.

In the rest of the paper, $E$ denotes a $(\varepsilon, \kappa)$-sample of $S$ and we provide asymptotic results when the sampling density increases, i.e. when $\varepsilon$ tends to 0 . As already mentioned, we consider $\kappa$ and the surface $S$ (and, in particular, the three quantities $C, A$ and $L$ ) to be fixed and not to depend on $\varepsilon$.

Several related sampling conditions have been proposed.

Amenta and Bern have introduced $\varepsilon$-samples [3] that fit locally the surface shape : the point density is high where the surface has high curvature or where the object or its complement is thin. However this definition is not appropriate for polyhedral surfaces since an $\varepsilon$-sample, as defined in [3], should have infinitely many points.

Erickson has introduced a notion of uniform sample that is related to ours but forbids two points to be too close [18]. Differently, our definition of a $(\varepsilon, \kappa)$-sample does not impose any lower bound on the minimal distance between two sample points.

In [5], Attali and Boissonnat use a slightly different definition of a $(\varepsilon, \kappa)$-sample. They assumed that for every point $x \in S$, the ball $B(x, \varepsilon)$ encloses at least one sample point and the ball $B(x, r)$ encloses $O\left(\frac{r^{2}}{\varepsilon^{2}}\right)$ sample points. With this sampling condition, they proved that the complexity of the Delaunay triangulation is $O\left(n^{1.8}\right)$ for general polyhedral surfaces and $O\left(n^{1.5}\right)$ for convex polyhedral surfaces. Our definition of a $(\varepsilon, \kappa)$-sample is slightly more restrictive since the facets need to be sampled independently of one another, which leads to add a few more sample points near the edges. However, the two conditions are essentially the same and our linear bound holds also under the slightly more general sampling condition of [5].

Golin and $\mathrm{Na}[20,21]$ assume that the sample points are chosen uniformly at random on the surface. The practical relevance of such a model is questionable since data are usually produced in a deterministic way.

\section{Preliminary results}

$S$ designates a polyhedral surface and $E \subset S$ a $(\varepsilon, \kappa)$-sample of $S$. Let $n(R)=|E \cap R|$ be the number of sample points in the region $R \subset S$. Let $n=|E|$ be the total number of sample points. We first establish two propositions relating $n(R)$ and $n$. We start with the following lemma:

Lemma 3 Let $F$ be a facet of $S$. For any $R \subset F$, we have:

$$
\frac{a(R)}{4 \pi \varepsilon^{2}} \leq n(R) \leq \frac{\kappa a(R \oplus \varepsilon)}{\pi \varepsilon^{2}}
$$



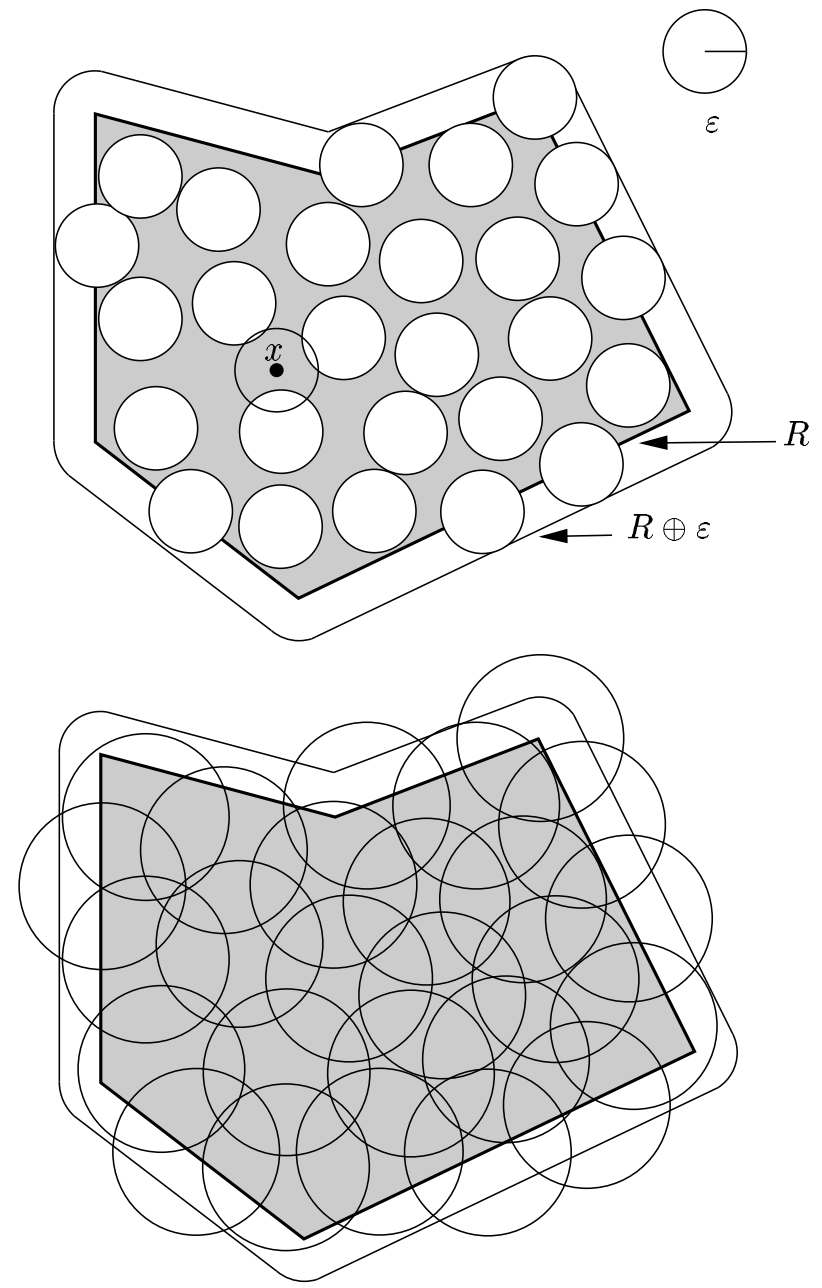

Figure 2: A maximal set of non-intersecting disks contained in $R \oplus \varepsilon$ and the corresponding covering of $R$ obtained by doubling the radii of the disks.

Proof. Let $\cup_{i=1}^{\lambda} D\left(m_{i}, \varepsilon\right)$ be a maximal set of $\lambda$ non-intersecting disks lying inside $R \oplus \varepsilon$. Because the set of disks is maximal, no other disk can be added without intersecting one of the $\lambda$ disks $D\left(m_{i}, \varepsilon\right)$. This implies that no point $x$ of $R$ is at distance greater than $2 \varepsilon$ from a point $m_{i}$ (see Figure 2). Therefore, $\cup_{i=1}^{\lambda} D\left(m_{i}, \varepsilon\right)$ is a packing of $R \oplus \varepsilon$ and $\cup_{i=1}^{\lambda} D\left(m_{i}, 2 \varepsilon\right)$ is a covering of $R$. Consequently:

$$
\frac{a(R)}{4} \leq \lambda \pi \varepsilon^{2} \leq a(R \oplus \varepsilon)
$$

$\mathrm{RR} \mathrm{n}^{\circ} 4453$ 
The disks lie in $R \oplus \varepsilon$. Therefore, the centers of the disks lie in $R$. By assumption, the disk $D\left(m_{i}, \varepsilon\right)$ contains at least one sample point and the disk $D\left(m_{i}, 2 \varepsilon\right)$ contains at most $\kappa$ sample points. Hence :

$$
\lambda \leq n(R) \leq \kappa \lambda
$$

and

$$
\frac{a(R)}{4 \pi \varepsilon^{2}} \leq n(R) \leq \frac{\kappa a(R \oplus \varepsilon)}{\pi \varepsilon^{2}}
$$

Proposition 4 Let $F$ be a facet of $S$. For any $R \subset F$, we have:

$$
n(R) \leq 4 \kappa \frac{a(R \oplus \varepsilon)}{A} n
$$

Proof. We first apply Lemma 3 to bound $n$ from below. Summing over the facets of $S$, we get :

$$
\frac{A}{4 \pi \varepsilon^{2}} \leq n
$$

We apply again Lemma 3 to bound $n(R)$ from above.

$$
n(R) \leq \frac{\kappa a(R \oplus \varepsilon)}{\pi \varepsilon^{2}}
$$

Eliminating $\varepsilon$ from the two inequalities yields the result.

Proposition 5 Let $F$ be a facet of $S$. Let $\Gamma \subset F$ be a curve contained in $F$. Let $k>0$. We have:

$$
n(\Gamma \oplus k \varepsilon) \leq \frac{(2 k+1)^{2}}{k} \kappa \frac{l(\Gamma)}{\varepsilon} \leq \frac{2(2 k+1)^{2}}{k} \sqrt{\pi} \kappa \frac{l(\Gamma)}{\sqrt{A}} \sqrt{n}
$$

Proof. Arguing as in the proof of Lemma 3, we see that the region $\Gamma \oplus k \varepsilon$ can be covered by $\frac{l(\Gamma)}{k \varepsilon}$ disks of radius $2 k \varepsilon$ centered on $\Gamma$ and contained in the supporting plane of $F$.

Applying Lemma 3 to a disk $R$ with radius $2 k \varepsilon$, we get:

$$
n(R) \leq \frac{\kappa \pi(2 k \varepsilon+\varepsilon)^{2}}{\pi \varepsilon^{2}}=(2 k+1)^{2} \kappa
$$

Therefore, we have :

$$
n(\Gamma \oplus k \varepsilon) \leq(2 k+1)^{2} \kappa \frac{l(\Gamma)}{k \varepsilon}
$$

From Equation 1, we get:

$$
\frac{1}{\varepsilon} \leq \frac{2 \sqrt{\pi}}{\sqrt{A}} \sqrt{n}
$$

Combining the two inequalities leads to the result. 


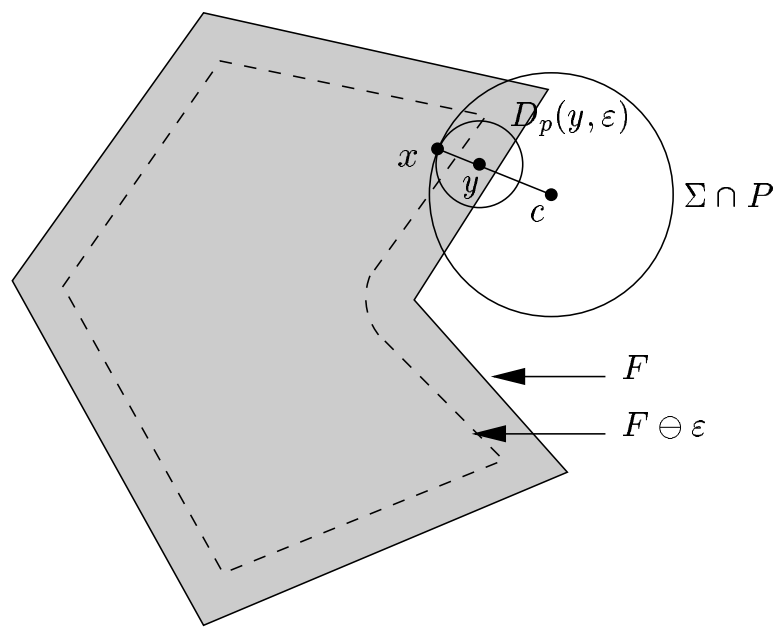

Figure 3: Assume $\Sigma$ is an empty sphere passing through a point $x \in F \ominus \varepsilon$ and intersecting the supporting plane of $F$ in a circle of radius greater than $\varepsilon$. Then, $\Sigma$ contains an empty disk $D_{p}(y, \varepsilon)$ centered on $F$.

Lemma 6 Let $x$ be a sample point in the $\varepsilon$-regular zone of $S$. Let $P$ be the supporting plane of the facet through $x$. Any empty sphere passing through $x$ intersects $P$ in a circle whose radius is less than $\varepsilon$.

Proof. The proof is by contradiction. Let $P$ be the supporting plane of $F$. Consider an empty sphere $\Sigma$ passing through $x$ and intersecting $P$ along a circle of radius greater than $\varepsilon$ (see Figure 3 ). Let $c$ be the center of this circle. Let $y$ be the point on the segment $[x c]$ at distance $\varepsilon$ from $x$. Because $x$ belongs to the $\varepsilon$-regular zone of $F, y \in F$. The empty sphere $\Sigma$ encloses the disk $D_{p}(y, \varepsilon)$. Therefore, $D_{p}(y, \varepsilon)$ is an empty disk of $P$, centered on $F$ and of radius $\varepsilon$, which contradicts our assumption.

\section{Counting Delaunay edges}

Let $S$ be a polyhedral surface and $E$ be a $(\varepsilon, \kappa)$-sample of $S$. The Delaunay triangulation of $E$ connects two points $p, q \in E$ iff there exists an empty sphere passing through $p$ and $q$. The edge connecting $p$ and $q$ is called a Delaunay edge. We will also say that $p$ and $q$ are Delaunay neighbours.

The number of edges $e_{p}$ and the number of tetrahedra $t_{p}$ incident to a vertex $p$ lying in the interior of the convex hull of $E$ are related by Euler formula

$$
t_{p}=2 e_{p}-4
$$


since the boundary of those tetrahedra is a simplicial polyhedron of genus 0 . Using the same argument, if $p$ lies on the boundary of the convex hull, we have:

$$
t_{p}<2 e_{p}-4
$$

By summing over the $n$ vertices, and observing that a tetrahedron has four vertices and an edge two, we get

$$
t<e-n \text {. }
$$

To bound the complexity of the Delaunay triangulation, it is therefore sufficient to count the Delaunay edges of $E$.

We distinguish three types of Delaunay edges : those with both endpoints in the $\varepsilon$-regular zone, those with both endpoints in the $\varepsilon$-singular zone and those with an endpoint in the $\varepsilon$-regular zone and the other in the $\varepsilon$-singular zone. They are counted separately in the following subsections,

We denote by $E_{s}$ the set of sample points in the $\varepsilon$-singular zone of $S$.

\subsection{Delaunay edges with both endpoints in the $\varepsilon$-regular zone}

In this section, we count the Delaunay edges joining two points in the $\varepsilon$-regular zone.

Lemma 7 Let $x$ be a sample point in the $\varepsilon$-regular zone and $F$ the facet that contains $x$. $x$ has at most $\kappa$ Delaunay neighbours in $F$.

Proof. By Lemma 6, any empty sphere passing through $x$ intersects $F$ in a circle whose radius is less than $\varepsilon$. Therefore, the Delaunay neighbours of $x$ on $F$ are at distance at most $2 \varepsilon$ from $x$. By assumption, the disk centered at $x$ with radius $2 \varepsilon$ contains at most $\kappa$ points of $E$.

Lemma 8 Let $x$ be a sample point in the $\varepsilon$-regular zone of a facet $F$. Let $F^{\prime} \neq F$ be another facet of $S$. $x$ has at most $\kappa$ Delaunay neighbours in the $\varepsilon$-regular zone of facet $F^{\prime}$.

Proof. Refer to Figure 4. $P$ and $P^{\prime}$ are the supporting planes of $F$ and $F^{\prime}, y^{\prime}$ is a Delaunay neighbour of $x$ in the $\varepsilon$-regular zone of $F^{\prime}$ and $\Sigma$ is an empty sphere passing through $x$ and $y^{\prime} . \Sigma$ intersects the planes $P$ and $P^{\prime}$ along two circles whose radii are respectively $r$ and $r^{\prime}$. By Lemma 6 , $r \leq \varepsilon$ and $r^{\prime} \leq \varepsilon$.

Let $M$ be the bisector plane of $P$ and $P^{\prime}$. Let $x^{\prime}$ and $y$ be the points symmetric to $x$ and $y^{\prime}$ with respect to $M$. Consider the smallest sphere passing through $x, x^{\prime}, y$ and $y^{\prime}$. This sphere intersects $P$ and $P^{\prime}$ in two disks of the same radius $r_{\min }$. We claim that $r_{\min } \leq \max \left(r, r^{\prime}\right)$. Indeed, let $c$ be the circumcenter of $\Sigma$, and $H_{x y^{\prime}}$ (resp. $H_{x^{\prime} y}$ ) be the bisector plane of $x$ and $y^{\prime}$ (resp. of $x^{\prime}$ and $y$ ). Observe that $v \in H_{x y^{\prime}} \cap H_{x^{\prime} y}$ and $c \in H_{x y^{\prime}}$. If $c \in H_{x y^{\prime}} \cap H_{x^{\prime} y}, r_{\min }=r=r^{\prime}$ and the claim is proved. Otherwise, $c$ must belong to one of the two open halfspaces limited by $H_{x^{\prime} y}$. If $c$ belongs to the halfspace that contains $x^{\prime}, \Sigma$ encloses $x^{\prime}$ and therefore $r_{\min } \leq r^{\prime}$ while in the second it encloses $y$ and $r_{\min } \leq r$. 


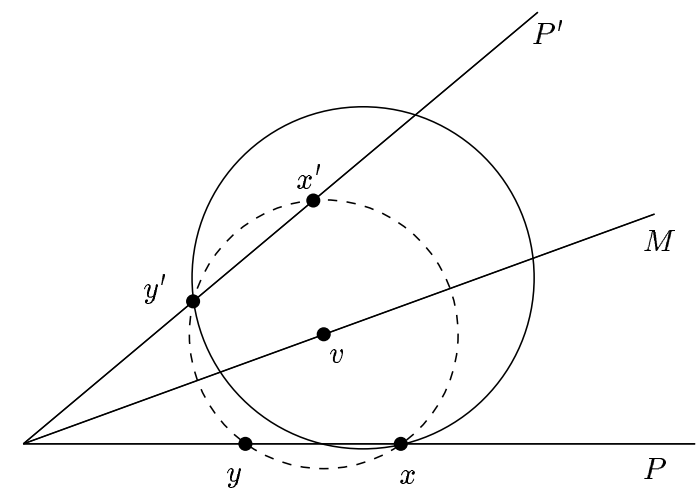

Figure 4: Any sphere passing through $x$ and $y^{\prime}$ intersects one of the two planes $P$ or $P^{\prime}$ in a circle whose diameter is at least $\left\|x^{\prime}-y^{\prime}\right\|$.

We therefore have :

$$
\frac{\left\|x^{\prime}-y^{\prime}\right\|}{2}=r_{\min } \leq \max \left(r, r^{\prime}\right) \leq \varepsilon
$$

and consequently:

$$
\left\|x^{\prime}-y^{\prime}\right\| \leq 2 \varepsilon .
$$

The Delaunay neighbours of $x$ in the $\varepsilon$-regular zone of $F^{\prime}$ lie in the disk $D_{p^{\prime}}\left(x^{\prime}, 2 \varepsilon\right)$. This disk contains at most $\kappa$ points of $E$.

Proposition 9 There are at most $\frac{C}{2} \kappa n$ Delaunay edges with both endpoints in the $\varepsilon$-regular zone of $S$.

Proof. The surface has $C$ facets. Therefore, by Lemmas 7 and 8 , a point $x$ in the $\varepsilon$-regular zone of $S$ has at most $C \kappa$ Delaunay neighbours.

\subsection{Delaunay edges with both endpoints in the $\varepsilon$-singular zone}

In this section, we count the Delaunay edges joining two points in the $\varepsilon$-singular zone.

Proposition 10 The number of Delaunay edges with both endpoints in the $\varepsilon$-singular zone is less than

$$
\frac{1}{2} 18^{2} \pi \kappa^{2} \frac{L^{2}}{A} n
$$

Proof. By Proposition 5, the number $\left|E_{s}\right|$ of sample points in the $\varepsilon$-singular zone is at most

$$
18 \sqrt{\pi} \kappa \frac{L}{\sqrt{A}} \sqrt{n}
$$

$\mathrm{RR} \mathrm{n}^{\circ} 4453$ 
Hence, the number of Delaunay edges in the $\varepsilon$-singular zone is at most $\frac{1}{2}\left|E_{s}\right| \times\left(\left|E_{s}\right|-1\right)<\frac{1}{2}\left|E_{s}\right|^{2}$.

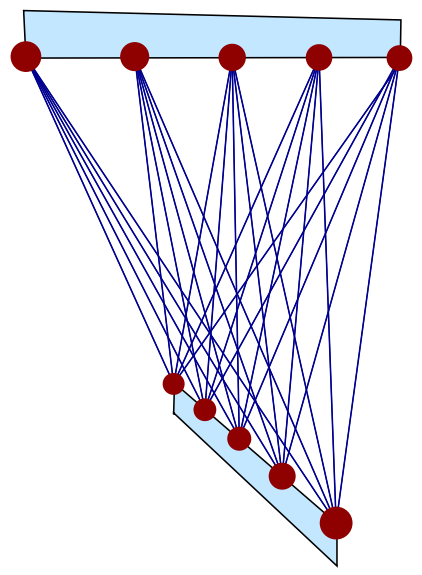

Figure 5: Example of a Delaunay triangulation of $m$ points having a quadratic number of edges. Even if such a configuration can occur for a subset of the sample points, the number of Delaunay edges involved in this configuration is $O(n)$.

\subsection{Delaunay edges joining the $\varepsilon$-regular and the $\varepsilon$-singular zones}

In this section, we count the Delaunay edges with one endpoint in the $\varepsilon$-regular zone and the other in the $\varepsilon$-singular zone.

We first introduce a geometric construction of independent interest that will be useful.

Let $P$ be a plane and $E_{s}$ be a set of points. We assign to each point $x$ of $E_{s}$ the region $V(x) \subset P$ consisting of the points $p \in P$ for which the sphere tangent to $P$ at $p$ and passing through $x$ encloses no point of $E_{s}$ (see Figure 6 ). In other words, if $R(p, x)$ denotes the radius of the sphere tangent to $P$ at $p$ and passing through $x$, we have:

$$
V(x)=\left\{p \in P: \forall y \in E_{s}, R(p, x) \leq R(p, y)\right\} .
$$

It is easy to see that the set of all $V(x), x \in E_{s}$, is a subdivision of $P$ we note $\mathcal{V}$ (see Figure 9). Let $\mathcal{P}_{x}$ be the paraboloid of revolution with focus $x$ and director plane $P$. The paraboloid $\mathcal{P}_{x}$ consists of the centers of the spheres passing through $x$ and tangent to $P$. Assume that the points $E_{s}$ are all located above plane $P$. If not, we replace $x$ by the point symmetric to $x$ with respect to $P$, which does not change $\mathcal{V}$. Let us consider the lower envelope of the collection of paraboloids $\left\{\mathcal{P}_{x}\right\}_{x \in E_{s}}$. Cell $V(x)$ is the projection of the portion of the lower envelope contributed by $\mathcal{P}_{x}$ (see Figures 6 and 9). 


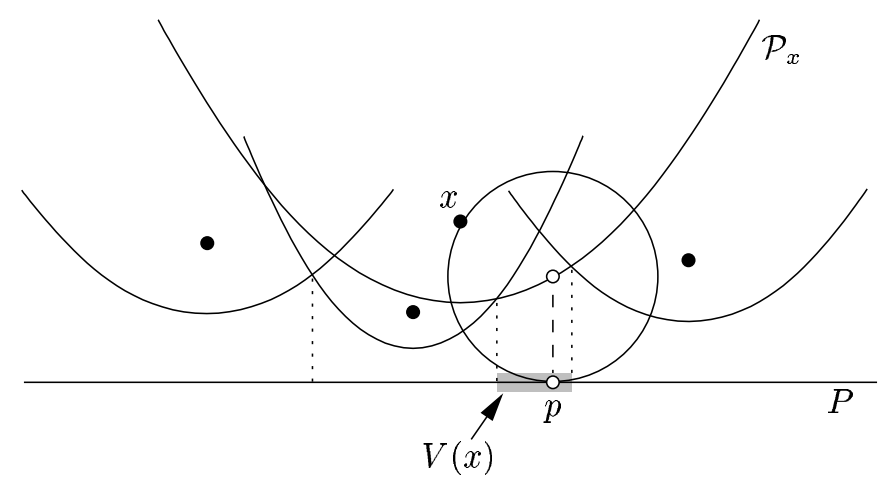

Figure 6: The cell $V(x)$ is the set of contact points between a plane $P$ and a sphere passing through $x$ and tangent to $P$. The part of the paraboloid $\mathcal{P}_{x}$ on the lower envelope of the paraboloids projects to the cell $V(x)$.

Consider the bisector $V(x, y)$ of $x, y \in E_{s}$, i.e. the points $p \in P$ such that $R(p, x)=R(p, y)$. $V(x, y)$ is the projection on $P$ of the intersection of the paraboloids $\mathcal{P}_{x}$ and $\mathcal{P}_{y}$. As easy computations can show, the bisector $V(x, y)$ of $x$ and $y$ is a circle or a line (considered as a degenerated circle). Let $H(x, y)$

$$
H(x, y)=\{p \in P: R(p, x) \leq R(p, y)\} .
$$

Since $V(x, y)$ is a circle, $H(x, y)$ is either a disk, in which case we rename it $D(x, y)^{+}$, or the complementary set of a disk $D(x, y)^{-}$. We therefore have

$$
V(x)=\bigcap_{y \in E_{s}, y \neq x} H(x, y)=\left(\cap D(x, y)^{+}\right) \backslash\left(\cup D(x, y)^{-}\right)
$$

It follows that the edges $E(x, y)$ of $V(x)$ are circle arcs that we call convex or concave wrt $x$ depending whether the disk $D(x, y)$ (whose boundary contains $E(x, y)$ ) is labelled + or - (see Figure 7). Observe that the convex edges of $V(x)$ are included in the boundary of the convex hull of $V(x)$.

Proposition 11 The number of Delaunay edges with one endpoint in the E-regular zone and the other in the $\varepsilon$-singular zone is at most :

$$
\left(1+450 \pi \kappa^{2} \frac{L^{2}}{A}\right) n
$$

Proof. Let $F$ be a facet of $S$ and $P$ the supporting plane of $F$. We bound the number of Delaunay edges with one endpoint in $E_{s}$ and the other in $E \cap(F \ominus \varepsilon)$, i.e. the number of Delaunay edges joining the $\varepsilon$-singular zone and the $\varepsilon$-regular zone of $F$.

We denote by $\mathcal{V}_{F}$ the restriction of the subdivision $\mathcal{V}$ introduced above to $F$, and, for $x \in E_{s}$, we denote by $V(x)$ the cell of $\mathcal{V}_{F}$ associated to $x$.

RR $n^{\circ} 4453$ 

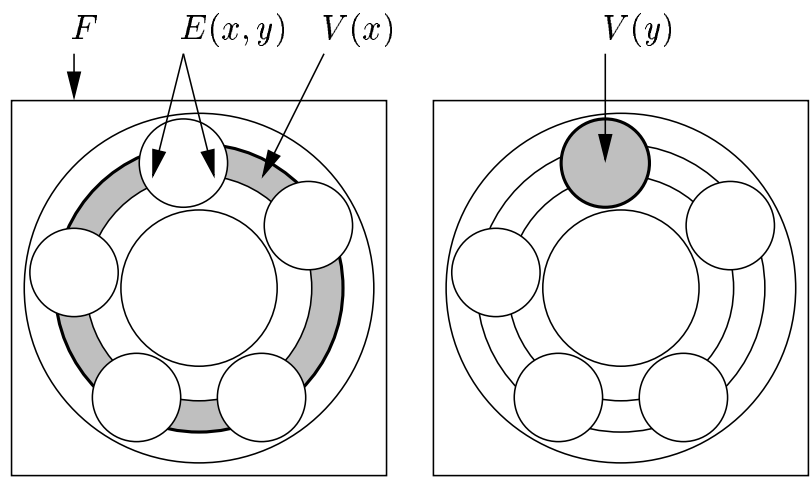

Figure 7: The bold edges are the convex edges of the shaded cells. The edge $E(x, y)$ which is concave wrt $x$ is convex wrt $y$. The convex edges of a cell lie on the boundary of its convex hull.

We first show that the Delaunay neighbours of $x$ that belong to the $\varepsilon$-regular zone of $F$ belong to $V(x) \oplus 2 \varepsilon$. Consider a Delaunay edge $(x f)$ with $x \in E_{s}, x \notin P$ and $f \in E_{s} \cap(F \ominus \varepsilon)$. Let $\Sigma$ be an empty sphere passing through $x$ and $f, v$ its center (see Figure 8). By Lemma 6, $\Sigma$ intersects $P$ in a circle whose radius $r$ is less than $\varepsilon$. For a point $c$ on the segment $[v x]$, we note $\Sigma_{c}$ the sphere centered at $c$ and passing through $x$. Because $\Sigma$ encloses $\Sigma_{c}, \Sigma_{c}$ is an empty sphere. For $c=v, \Sigma_{c}$ intersects $P$. For $c=x, \Sigma_{c}$ does not intersect $P$. Consequently, there exists a position of $c$ on $[v x]$ for which $\Sigma_{c}$ is tangent to $P$. Let $p=\Sigma_{c} \cap P$ for such a point $c$. We have $p \in V(x)$ and $\|p-f\| \leq 2 r \leq 2 \varepsilon$. Hence, $f \in V(x) \oplus 2 \varepsilon$. Now, let us consider a Delaunay edge $(x f)$ with $x, f \in E_{s} \cap P$. Applying Lemma 6 leads to $f \in V(x) \oplus 2 \varepsilon$.

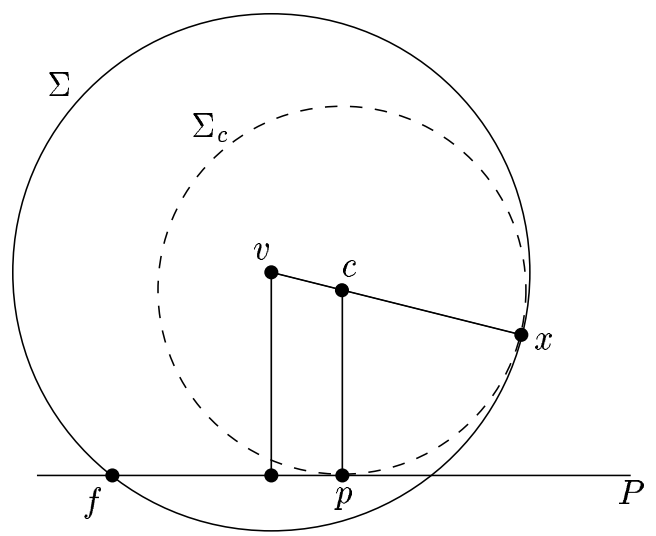

Figure 8: Every sphere $\Sigma$ passing through $x$ and $f \in P$ contains a sphere $\Sigma_{c}$ passing through $x$ and tangent to $P$. 
Let $N_{F}$ be the number of Delaunay edges between $E_{s}$ and $F \ominus \varepsilon$. We have, using the fact that $\mathcal{V}_{F}$ is a subdivision of $F$ and Proposition 5 :

$$
\begin{aligned}
N_{F} & \leq \sum_{x \in E_{s}} n(V(x) \oplus 2 \varepsilon) \\
& \leq n(F)+\sum_{x \in E_{s}} n(\partial V(x) \oplus 2 \varepsilon) \\
& \leq n(F)+25 \sqrt{\pi} \kappa \frac{1}{\sqrt{A}} \sqrt{n} \sum_{x \in E_{s}} l(\partial V(x))
\end{aligned}
$$

Let us bound $\sum_{x \in E_{s}} l(\partial V(x))$. Given a cell $V(x)$, we bound the length of its convex edges. By summing over all $x \in E_{s}$, all edges in $\mathcal{V}_{F}$ will be taken into account.

The convex edges of $x$ are contained in the boundary of the convex hull of $V(x)$. Since $V(x) \subset$ $F$, the length of the boundary of the convex hull of $V(x)$ is at most the length of $\partial F$. Consequently:

$$
\sum_{x \in E_{s}} l(\partial V(x)) \leq l(\partial F) \times\left|E_{s}\right|
$$

Since, by Proposition 5, $\left|E_{s}\right| \leq 18 \sqrt{\pi} \kappa \frac{L}{\sqrt{A}} \sqrt{n}$, we have:

$$
N_{F} \leq n(F)+450 \pi \kappa^{2} \frac{l(\partial F) \times L}{A} n
$$

By summing over all the facets, we conclude that the total number of Delaunay edges with one endpoint in the $\varepsilon$-regular zone and the other in $\varepsilon$-singular zone is at most :

$$
\left(1+450 \pi \kappa^{2} \frac{L^{2}}{A}\right) n
$$

\subsection{Main result}

We sum up our results in the following theorem :

Theorem 12 Let $S$ be a polyhedral surface and $E$ a $(\varepsilon, \kappa)$-sample of $S$ of size $|E|=n$. The number of edges in the Delaunay triangulation of $E$ is at most :

$$
\left(1+\frac{C \kappa}{2}+612 \pi \kappa^{2} \frac{L^{2}}{A}\right) n
$$

It should be observed that the bound does not depend on the relative position of the facets (provided that their relative interiors do not intersect). Notice also that the bound is not meaningful when $A=0$, which is the case of the quadratic example in Figure 5.

$\mathrm{RR} \mathrm{n}^{\circ} 4453$ 


\section{Conclusion}

We have shown that, under a mild sampling condition, the Delaunay triangulation of points scattered over a fixed polyhedral surface or any fixed pure piece-wise linear complex has linear complexity. Therefore, we (partially) answered an old question of Boissonnat [8]. Our sampling condition does not involve any randomness (as in the work by Golin and $\mathrm{Na}$ [20]) and is less restrictive than Erickson's one [18].

Although the sampling condition has been expressed in a simple and intuitive way, the linear bound holds under a more general setting. Indeed, all we need for the proof is to subdivide the surface in two zones, an $\varepsilon$-regular zone where one can apply Lemma 6 and an $\varepsilon$-singular zone containing $O(\sqrt{n})$ points.

As mentionned in the introduction, Erickson has shown that the Delaunay triangulation of $n$ points distributed on a cylinder may be quadratic. To understand where our analysis fails for such an example, one has to remember that our proof relies on Lemma 6 which states that empty balls intersect polyhedral surfaces in disks whose area is smaller than $\pi \varepsilon^{2}$, which is not the case anymore in Erickson's example.

The main open question is of course to consider the case of smooth surfaces. The $O(n \sqrt{n})$ lower bound obtained by Erickson for cylinders show that a linear bound does not hold for arbitrary surfaces. We conjecture that, for generic surfaces, the complexity of the Delaunay triangulation is

still linear. We say that a surface $S$ is generic if 1 . its maximal balls intersect $S$ at a finite number of so-called contact points, and 2. the intersection of $S$ with the union of the maximal balls with only one contact point form a set of curves of finite length on $S$. In particular, generic surfaces cannot contain spherical nor cylindrical pieces.

\section{References}

[1] N. Amenta, M. Bern, and M. Kamvysselis. A new Voronoi-based surface reconstruction algorithm. In Proc. SIGGRAPH '98, pages 415-412, July 1998.

[2] N. Amenta, S. Choi, T.K. Dey, and N. Leekha. A simple algorithm for homeomorphic surface reconstruction. In Proc. 16th Annu. ACM Sympos. Comput. Geom., pages 213-222, 2000.

[3] Nina Amenta and Marshall Bern. Surface reconstruction by voronoi filtering. Discrete Comput. Geom., 22:481-504, 1999.

[4] Nina Amenta and Ravi Krishna Kolluri. Accurate and efficient unions of balls. In Proc. 16th Annu. ACM Sympos. Comput. Geom., pages 119-128, 2000.

[5] Dominique Attali and Jean-Daniel Boissonnat. Complexity of the delaunay triangulation of points on polyhedral surfaces. Technical Report RR-4232, INRIA, July 2001. http:// www-sop.inria.fr/rapports/sophia/RR-4232.html.

[6] F. Bernardini, C. L. Bajaj, J. Chen, and D. R. Schikore. Automatic reconstruction of 3D CAD models from digital scans. Internat. J. Comput. Geom. Appl., 9:327-369, 1999. 
[7] F. Bernardini, J. Mittleman, H. Rushmeier, C. Silva, and G. Taubin. The ball-pivoting algorithm for surface reconstruction. IEEE Trans. on Visualization and Computer Graphics, 5(4):349$359,1999$.

[8] J. D. Boissonnat. Geometric structures for three-dimensional shape representation. ACM Transactions on Graphics, 3(4):266-286, October 1984.

[9] J.-D. Boissonnat and F. Cazals. Smooth surface reconstruction via natural neighbour interpolation of distance functions. In Proc. 16th Annu. ACM Sympos. Comput. Geom., pages 223-232, 2000 .

[10] Jean-Daniel Boissonnat and Frédéric Cazals. Natural neighbour coordinates of points on a surface. Computational Geometry - Theory and Application, 19(2-3):87-220, 2001.

[11] Jean-Daniel Boissonnat and Mariette Yvinec. Algorithmic Geometry. Cambridge University Press, UK, 1998. Translated by Hervé Brönnimann.

[12] T. M. Chan, J. Snoeyink, and C. K. Yap. Primal dividing and dual pruning: Output-sensitive construction of 4-d polytopes and 3-d Voronoi diagrams. Discrete Comput. Geom., 18:433454, 1997.

[13] L. P. Chew. Guaranteed-quality mesh generation for curved surfaces. In Proc. 9th Annu. ACM Sympos. Comput. Geom., pages 274-280, 1993.

[14] S. Choi and N. Amenta. Delaunay triangulation programs on surface data. In Proc. of 13th ACM-SIAM Symposium on Discrete Algorithms, San Francisco, CA, January 2002.

[15] R. A. Dwyer. Higher-dimensional voronoi diagram in linear expected time. Discrete Comput. Geom., 6:343-367, 1991.

[16] R. A. Dwyer. The expected number of $k$-faces of a voronoi diagram. Internat. J. Comput. Math., 26(5):13-21, 1993.

[17] H. Edelsbrunner. Deformable smooth surface design. Discrete Comput. Geom., 21:87-115, 1999.

[18] Jeff Erickson. Nice point sets can have nasty Delaunay triangulations. In Proc. 17th Annu. ACM Sympos. Comput. Geom., pages 96-105, 2001.

[19] Jeff Erickson. Dense point sets have sparse delaunay triangulations. In Proc. of the 13th Annual ACM-SIAM Symposium on Discrete Algorithms, 2002. To appear. http://www. cs . ust . $\mathrm{hk} / \mathrm{tcSC} / \mathrm{RR} /$.

[20] Mordecai J. Golin and Hyeon-Suk Na. On the average complexity of 3d-voronoi diagrams of random points on convex polytopes. In Proc. 12th Canad. Conf. Comput. Geom., 2000. http://www.cs.ust.hk/tcsc/RR/. 
[21] Hyeon-Suk Na Mordecai J. Golin. The probabilistic complexity of the voronoi diagram of points on a polyhedron. In Proc. ACM Sym. on Computational Geometry, 2002. To appear.

[22] D.J. Sheehy, C.G. Armstrond, and D.J. Robinson. Computing the medial surface of a solid from a domain delaunay triangulation. In Proc. 3rd Symposium on Solid Modeling and Applications, pages 201-212, May 1995.

[23] Andrew Thall, Stephen Pizer, and Tom Fletcher. Deformable solid modeling using sampled medial surfaces: A multiscale approach. Technical Report TR00-005, January 2000. http: //citeseer.nj.nec.com/thalloodeformable.html. 


\section{Contents}

$\begin{array}{llr}1 & \text { Introduction } & 3\end{array}$

2 Definitions and notations $\quad 4$

2.1 Voronoi diagrams and Delaunay triangulations . . . . . . . . . . . . . . . 4

2.2 Notations . . . . . . . . . . . . . . . . . . . . . . . . 4

2.3 Polyhedral surfaces ......................... 5

2.4 Sample ........................... 5

3 Preliminary results $\quad 6$

4 Counting Delaunay edges $\quad 9$

4.1 Delaunay edges with both endpoints in the

4.2 Delaunay edges with both endpoints in the

$\varepsilon$-singular zone . . . . . . . . . . . . . . . . . . . . . 11

4.3 Delaunay edges joining the $\varepsilon$-regular and the $\varepsilon$-singular zones . . . . . . . . . 12

4.4 Main result ... . . . . . . . . . . . . . . . . . . . 15

5 Conclusion $\quad 16$

$\mathrm{RR} \mathrm{n}^{\circ} 4453$ 

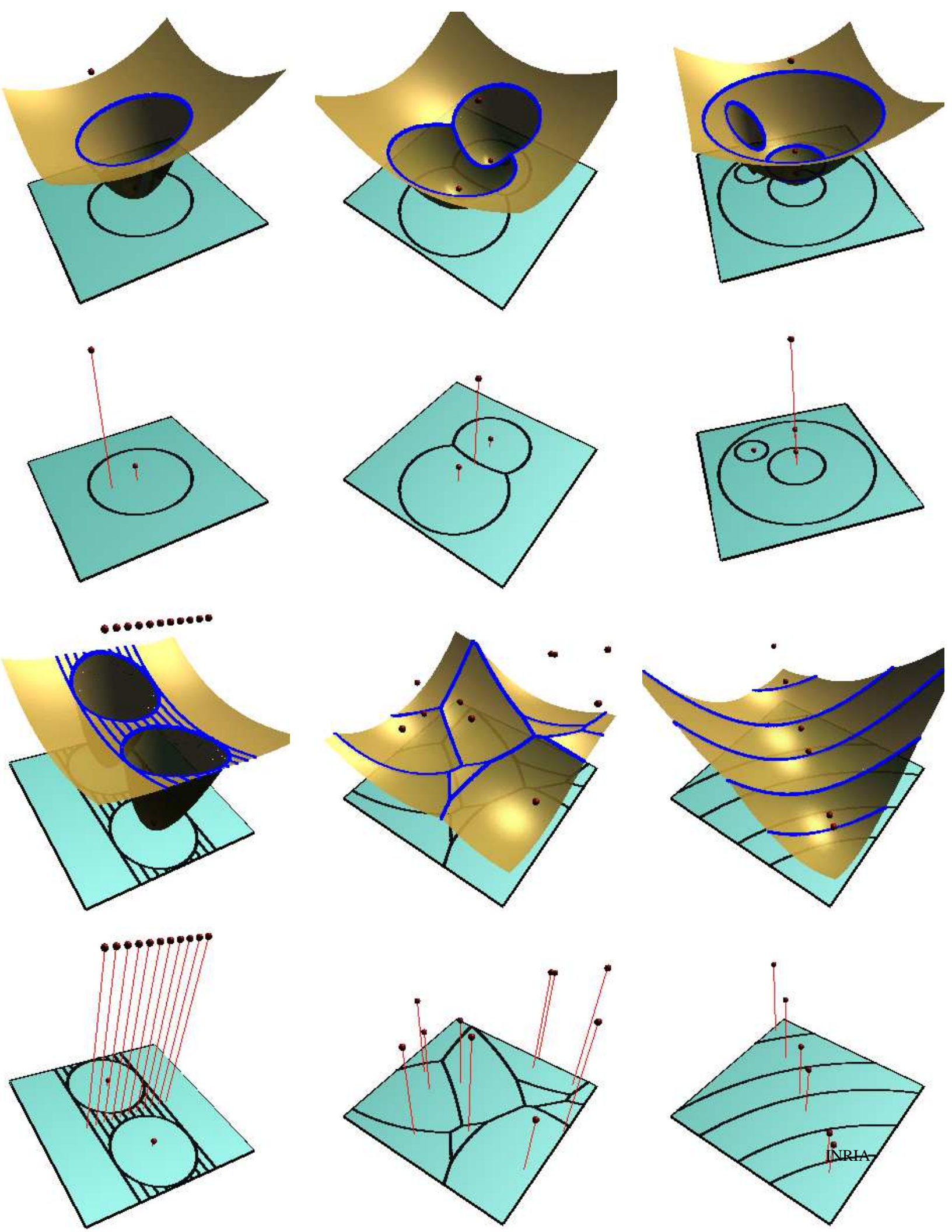

Figure 9: Decomposition of a facet $F$ into cells for different set of points $E_{s}$. The lower envelope of the paraboloid $\left\{\mathcal{P}_{x}\right\}_{x \in E_{s}}$ has been represented. The red spheres represent the points of $E_{s}$ and the red lines materialize the projection of the points of $E_{s}$ on the plane $P$. The bisector of two points is a circle. The projection of $x$ on $P$ do not belong necessary to its cell. The decomposition of $F$ can have a quadratic number of edges. 


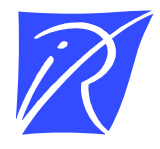

Unité de recherche INRIA Sophia Antipolis 2004, route des Lucioles - BP 93 - 06902 Sophia Antipolis Cedex (France)

Unité de recherche INRIA Lorraine : LORIA, Technopôle de Nancy-Brabois - Campus scientifi que 615, rue du Jardin Botanique - BP 101 - 54602 Villers-lès-Nancy Cedex (France)

Unité de recherche INRIA Rennes : IRISA, Campus universitaire de Beaulieu - 35042 Rennes Cedex (France)

Unité de recherche INRIA Rhône-Alpes : 655, avenue de l'Europe - 38330 Montbonnot-St-Martin (France)

Unité de recherche INRIA Rocquencourt : Domaine de Voluceau - Rocquencourt - BP 105 - 78153 Le Chesnay Cedex (France)

Éditeur

INRIA - Domaine de Voluceau - Rocquencourt, BP 105 - 78153 Le Chesnay Cedex (France)

http://www.inria.fr

ISSN 0249-6399 